\title{
Research on New Smart City from the Perspective of Blockchain Technology
}

\author{
Dr. Li Bahao \\ Jiujiang University School of Economics and Management
}

Keywords: blockchain; smart; city

\begin{abstract}
The country is now experiencing rapid economic development, while the city is also faced with many problems. In recent years, China has invested heavily in manpower and material resources in smart cities, and has made corresponding plans by setting the first batch of pilot smart cities. The so-called smart city refers to the use of various advanced technologies, including cloud computing and the Internet of Things, to analyze the data of various industries in urban development. Therefore, it provides certain data support for the work of relevant government functional departments and effectively promotes urban development. Based on this, this paper focuses on the foundation of a new smart city supported by blockchain technology. By expounding the function of blockchain technology and the practical problems that it solves, the application of blockchain technology in new smart cities is analyzed. Finally, it is hoped to strengthen everyone's further understanding with this paper and attach great significance to it.
\end{abstract}

\section{Introduction}

The urban development is an important reflection of the economic development of the country. However, there are many problems in the current urban development, and the problem is gradually shortened from the stage of germination to the time of deterioration. Such unscientific distribution of social resources and increasingly serious environmental problems have seriously threatened people's daily life and the advancement of the city. Under such circumstances, the development of smart cities is put forward. Smart cities use a variety of advanced technologies in urban planning and management, which promotes the smooth urban development by pursuing a green, harmonious and open development concept.

\section{Conceptual analysis of new smart cities}

The concept of a new smart city is a up-to-day idea for urban construction proposed by contemporary scholars based on the needs of various industries in the city and supported by relevant technologies. Actual needs of users are analyzed on the basis of the corresponding data, and then the data is adjusted in return according to user needs. In the end, it is enabled to achieve the important goal of reducing urban resource waste and economic consumption as well as optimizing the economic supply side structure ${ }^{[1]}$. For instance, in a new smart city, electricity planning can be performed via corresponding technology. By collecting the amount of electricity used by the user over a period of time, not only the power consumption of all users is summed up, but the total power supply of a city during this period is also obtained. Then, in contrast with the supply standard of urban power resources, it is possible to know whether the supply of urban power meets the standards. If there is a power outage, it is able to know how much is exceeded. This can be specific to individual users to learn about urban areas that exceed power supply standards, which effectively control the power supply in this area and ensure the accuracy of its power supply. In order to specify the power supply to individual users, it is necessary to have other data of feedback information from users to analyze. For example, based on the operating data of heating facilities of one user, it is likely to preliminarily determine the amount of electricity used by an individual user in heating. According to this method, the research and analysis of the user's other power consumption can obtain the total amount of power consumption of the user for a period of time, thereby achieving effective control. 


\section{Blockchain technology features}

\section{(1) Distributed storage function}

Distributed storage is the most basic function in blockchain technology. The blockchain system structure is divided into a network interface, a distributed database, and a processing center. The processing center, as a terminal part, has a corresponding network interface. The distributed database is connected to the processing center through these network interfaces to form a distributed form. In this form, all distributed databases are stored separately. However, due to the role of the network, the processing center can still schedule it ${ }^{[2]}$. In addition, through the data scheduling function of the processing center, all data scheduled to the processing center will be classified and stored according to the specific characteristics of the information, which can effectively avoid data confusion.

(2) Big data technology function

In a way, blockchain technology has evolved from big data technology with multiple functional features of big data technology. For example, the scheduling function of the blockchain processing center is to use the feature learning function to deeply analyze the acquired data, and then obtain the corresponding information including semantic and connotation features. Due to the role of deep mining information, other information is used to refine the application information of individual users on a certain resource. Blockchain technology will dig deeper into all the data and derive the logical relationship between various information, thus further determining the relationship between the weights. It can be seen that under the influence of big data technology, blockchain technology fully demonstrates the characteristics of intelligence.

(3) Point-to point transmission

The role of data scheduling in blockchain technology does not only apply to distributed databases and processing centers. In each of the distributed databases, the corresponding data can also be transmitted. It can be seen that the data transmission of the technology realizes the functional characteristics of point-to-point transmission of data information ${ }^{[3]}$. For the transmission of point-topoint information between distributed databases in actual needs, if an operating management company has data needs, specific instructions can be formed and presented on the corresponding distributed database. In this way, the management company in the distributed databases can deliver the corresponding information to the management company of the city operation according to the instruction information. If the management company in the distributed database considers that the data information does not need to be transmitted, it can reject the instruction.

(4) Consensus mechanism

The consensus mechanism is the main application technology in blockchain technology. Although it maintains the independence of information in the overall structure of the system, in the operation of the system, all information actions are carried out centering on one target. It is assumed that reducing energy consumption is one of the important functions of this mechanism, which distributed databases and processing centers will consider as the main target during operation. In other words, the processing center analyzes the energy supply over a period of time based on standard values. All distributed databases will deliver energy supply information to the processing center during this period. Then, in accordance with the final analysis of the processing center, whether the current urban energy supply exceeds the standard is effectively determined. Besides, it can also be compared with the standard values to know exactly how much energy is wasted during that time period, thus indicating the direction for the development of the governance plan.

\section{Problems solved by blockchain}

The application of blockchain technology in the city intelligent big data platform effectively deals with many existing problems. For example, using the features in which the powers and responsibilities are distributed in a decentralized manner, the original service ports and many of the information therein are respectively allocated in different nodes, which greatly lowers the total operating capital investment of the system; The peer-to-peer node function of this technology can 
ensure that the node can maintain its operation in addition to the public service, which not only reduces the difficulty of research and development, but also improves the overall efficiency of the system; In addition, smart contracts in this technology are applied at all aspects. When accessing new information and operating modes in the system, it is possible to set up a smart contract instead of rebuilding the data information structure and storage mode, which helps improve efficiency and makes it more convenient and faster.

The big data system of smart city construction from the perspective of blockchain includes data sources, regulatory access services, and operators. Blockchain technology is of great importance in smart city big data systems, as it has multiple functions such as providing information and managing information [4]. The system adopts a mode of synchronizing data information so as to achieve the purpose of effectively ensuring information input, output and storage efficiency. What's more, in the entire big data system, the supervisory control is the key. Decentralization is the main feature of the blockchain. Its supervisory control method is to supervise and control through the nodes in the chain. For instance, due to the high requirements of intelligent transportation, the application of this technology in intelligent transportation is reflected in the scientific preservation and application of data related to various subjects such as road management, climate, and traffic vehicles. The vehicle data information is provided by the traffic management department or through the sharing of users of related applications such as the Gaode map. The climate information data is provided by the meteorological management department, or it is learnt from actual management measures of the roads by relevant government departments. All kinds of data information mentioned above must be analyzed and processed by the participants in the data. Only in this way can the data be transmitted to the system by the node, which can be provided to the relevant users for inquiry and application.

\section{Specific application of blockchain technology in the development concept of new smart city}

\section{(1) Data sharing}

The point-to-point transmission of information in the blockchain technology can realize information sharing, which is implemented by the following four aspects.

\section{Decentralization}

In the new smart city, the decentralization of blockchain technology is a new form of deployment, which is achieved through the POW mechanism. Moreover, the information sharing module is used to replace the processing center. Due to the complexity of the current network environment, there are various ways of attacking the network. As the processing center structure of the terminal system, it is possible to be attacked regardless of the protection means. The important role of this mechanism is that it can effectively circumvent this situation if the information sharing module is utilized. This is mainly because the information sharing module has the characteristics of timeliness. At the same time, it can encrypt the corresponding information, and the information can be shared among the modules at the fastest speed. In such a case, if the user wants to view the corresponding information, the user's identity must be verified, and the permission of the relevant data source enterprise is also need. Therefore, it owns higher security.

2. Data that cannot be tampered with

The most critical role of the trust mechanism is that data cannot be tampered with arbitrarily. In exchanging data information, the data information cannot be changed without the permission of both parties. Therefore, it can effectively avoid some fraud cases and improve the security of the system ${ }^{[5]}$. Additionally, for the consensus mechanism described above, the trust mechanism is its deep manifestation, which is realized by the HashCash algorithm. This method can verify the reliability of the data information results. To a certain extent, the trust between the two parties is enhanced.

3. Transparent exchanging process

Energy data not only reflects the actual energy application of users, but also reflects the economic benefits of energy companies to some extent. When users increase energy consumption, energy companies need to invest more in production to ensure energy supply. It can be seen that there is a 
certain correspondence between energy data information and account information of energy companies. Blockchain technology has a function of the shared ledger service. In order to effectively avoid the fraud of energy data information, errors or omissions, the transparency of data information exchange can be effectively guaranteed with the help of the shared ledger service. In the case of data sharing, data source companies use the records in the shared ledger or other information to verify the reliability of the data.

4. Source locating

In the past, the process of applying blockchain technology often caused difficulties in determining the ownership of information, which led to many disputes. With the advancement of technology, in the new smart city, the important role of source locating is realized. In detail, the logic of the technology is based on data ownership and combined with big data technology to analyze all data information. The content of the data information, such as the weights of the source data, is determined by tracking the change of the data information. In this way, if there is a dispute, the ownership of the information can be determined based on the weights of the source data and the tracking record. In addition, it is not possible to compare the weight levels for data information at different time periods. Therefore, in order to enable the synchronization of the compared information to be effectively guaranteed, in the source locating of the data information, a function of adding a timestamp is required. The corresponding time and date are provided to the user, so that the synchronization of the information is guaranteed.

(2) Blockchain guarantees information security in new smart cities

In recent years, many information leakage incidents in China and abroad have reflected the unreliability of centralized data storage. In the absence of a trust system, local data information storage brings a sense of distrust and crisis. In new smart cities, blockchain technology with decentralized storage has gradually emerged.

The difference in blockchain lies in the way data is stored. Decentralized distributed storage is the main method used by this technology. It distributes the data information to have multiple nodes and stores it in a decentralized form by encrypting the data information. In conjunction with the corresponding consensus agreement, the contract will be initiated and implemented under certain conditions. The content in the contract is publicly displayed for each node. Since the data is stored in different locations, even if one of the networks is in a state of being paralyzed and unable to work, the user can still obtain data information from other nodes ${ }^{[6]}$. With smart contracts, it is possible to pre-define rules related to smart city data information sharing, such as the visible range of data information resources and the exchange mode of data information. The use of signature encryption ensures that documents are not tampered with, thereby maximizing the security and reliability of data information. The relevant departments have different understanding in terms of data sharing and data information subjects. Consequently, different read and write permissions are assigned to different nodes for different data information sharing ranges. The vast amount and variety of data information in new smart cities, coupled with the corresponding IoT facilities, will generate a large amount of data. In this case, the use of the blockchain can be developed in a customized manner in combination with its development space and security needs.

(3) Blockchain contributes to government big data in new smart cities

At present, the government big data platforms usually use the solutions provided by platform vendors to manage data information in a centralized and intensive manner. The blockchain technology can use P2P nodes to distribute the data information in the centralized server in a distributed manner on the nodes of the corresponding users. This not only greatly reduces the difficulty of developing data information platform technology, but also lowers the cost of system development and future operating cost. At the same time, it effectively facilitates the ability to process dedicated data information. In the peer node, each node stores the same data information, can perform related operations separately, and plays the role of mutual supervision ${ }^{[7]}$. Faced with the new data information model and business model, a consensus protocol is introduced. It only needs to use the software to deploy the consensus protocol, which does not need to re-create the current interface 
information structure and information storage structure, so the scalability of the system is improved to some extent. In the relevant government business, if the requirements for confidentiality of information are not very high, blockchain technology based on public chain identity can be used to set up a consensus mechanism and monitor smart contracts. Although the information in the chain is invisible, it is public in the network, which provides a powerful guarantee for the realization of technologies such as identification. In addition, private data information related to the identity blockchain, such as credit conditions and biometrics, can also be stored in this blockchain. The relevant information in the identity blockchain only displays evidence that proves the corresponding nature of its identity information, and does not show details. The data related to it will be selectively presented in a transparent manner, which effectively guarantees the security of private information. For government departments that have high requirements on the confidentiality of data information, such as public security, they can use the private chain to exchange data information. By running in the internal network, the security of data transmission and the mutual supervision of each node are ensured, so is traceability of data information. According to the actual needs of the information receiving party, the information owner and the data security, each relevant department can create its own private chain to transmit the corresponding business data information.

\section{Conclusion}

In recent years, blockchain from the emerging to the vigorous development, has inspired relevant professionals to continue exploring its usage. The technology was initially got in touch with by only a few people. Afterwards, it evolves into a new and popular technology that is applied in various fields. For example, it receives favorable results in the development of government-related work and community management. The construction of smart cities is closely related to blockchain technology. In fact, the application of blockchain technology to the construction of smart cities is conducive to transforming the role of government departments into project supervisors. This allows participating subjects to better serve the community and create more economic benefits. In another sense, the role of government departments has changed from past dominance to present supervisor. The participation of people in urban construction not only reduces capital investment, but also improves the efficiency of government departments. At the same time, it can enhance the service quality and promote the urban development more quickly and steadily.

\section{References}

[1] Liang Yueting. Research on building a big data platform for smart city based on blockchain[J]. Technology and Economic Guide, 2019, 27(28): 24.

[2] Duan Yingjun. Supply chain innovation in the new era: the conception of smart supply chain holographic ecosphere construction supported by blockchain as the core technology[J]. China Economist, 2019(08):11-13+15.

[3] Liu Chunming. Discussion on the application of blockchain technology in the construction of new smart cities [J]. Intelligent City, 2019, 5 (12): 28-29.

[4] Li Beijia, Lin Baocheng, Chen Yi, Shen Yan, Xu Ziyu. The application of blockchain technology in the construction of smart broadcasting [J]. Cable Television Technology, 2019 (06): 87-90.

[5] Guan Zhigui, Tian Xuebin, Kong Youhua. Research on the ecological value realization path of Xiong'an new district based on blockchain technology[J]. Journal of Hebei University of Economics and Business,2019,40(03):77-86.

[6] Building a financial highland for blockchain and a brand for "smart city" _ Documentary on the construction of the financial technology business service new district of Industrial and Commercial Bank of China, Hebei Xiong'an Branch[J]. Journal of Hangzhou Institute of 
Financial and Economics, 2019(04):19-21.

[7] Yang Deqin, Yue Aobo, Yang Ruijia. Management research on information integration of intelligent projects_-Based on the application of blockchain technology [J]. Construction Economy, 2019,40(02):80-85.

[8] Li Haolun. Research on the construction of big data-based platform of smart city based on blockchain[J]. Technology Wind, 2018(36):106.

[9] Chen Ping. Smart city ecological solution that will help the development of the Pearl River Delta [J]. China Public Security, 2018 (12): 60-62.

[10]. Foshan Chancheng leverages the blockchain to create an "intelligent honesty city" [J]. Information for Deciders Magazine, 2018 (44): 18-19.

[11] Yang Junfeng. Application research of blockchain technology in the construction of new smart city[J]. Telecom World, 2018(10):36-37.

[12] Wang Zeyu. Research on the construction of smart city based on blockchain-based big data platform [J]. Science and Technology Innovation Herald, 2018, 15 (24): 38-41. 